\title{
LncRNA CRNDE acts as a ceRNA and regulates AML cell proliferation and apoptosis via miR136- 5p/MCM5 axis
}

Chen Liu

Chongqing Medical University

Liang Zhong

Chongqing Medical University

Chenlan Shen

Chongqing Medical University

Xuan Chu

Chongqing Medical University

Xu Luo

Chongqing Medical University

Lihua Yu

Chongqing Medical University

Jiao Ye

Chongqing Medical University

\section{Ling Xiong}

Chongqing Medical University

Wenran Dan

Chongqing Medical University

Jian Li

Chongqing Medical University

Beizhong Liu ( $\nabla$ liubeizhong@cqmu.edu.cn )

https://orcid.org/0000-0001-8360-6646

Primary research

Keywords: acute myeloid leukemia, Long non-coding RNA, CRNDE, miR-136-5P, MCM5

Posted Date: September 3rd, 2020

DOI: https://doi.org/10.21203/rs.3.rs-62224/v2 
License: (c) (i) This work is licensed under a Creative Commons Attribution 4.0 International License. Read Full License 


\section{Abstract}

Background Increasing evidence demonstrated that long noncoding RNAs (IncRNAs) act as important factors in the regulation of cell processes and tumorigenesis. The long-noncoding RNA colorectal neoplasia differentially expressed (CRNDE) gene has been found to be related to several types of cancer. Although CRNDE is highly expressed in AML, its mechanism of action in acute myeloid leukemia (AML) is unknown. Methods The expression levels of CRNDE and miR-136-5p mRNAs were measured by quantitative real-time PCR. The effects of CRNDE knockdown on cell proliferation was assessed by the CCK8 assay, while apoptosis and cell cycle distribution were analyzed by flow cytometry. The expression of proteins related to cell cycle, cell apoptosis and MCM5 were analyzed by Western blotting. The luciferase reporter assay was used to confirm the interaction between CRNDE and miR-136-5p and between MCM5 and miR-136-5p in AML. The RNA immunoprecipitation assay was used to verify whether CRNDE exists in the miRNA mediated RISC complex. Results In this study, we used GEPIA database to confirm that CRNDE expression was significantly upregulated in AML samples. The silencing of CRNDE inhibited AML cells' proliferation ability, increased AML cells' apoptotic rate and arrested AML cells at the G1 phase of the cell cycle. Mechanistically, CRNDE served as a competing endogenous RNA (ceRNA) for miR-136-5p and upregulated MCM5 expression by sponging miR-136-5p. In addition, rescue assays revealed that the effects of CRNDE knockdown could be reversed by miR-136-5p inhibitors in AML cells. Conclusion Our results demonstrate that the CRNDE-miR-136-5p-MCM5 axis modulates AML progression and provide a new regulatory network of CRNDE in AML.

\section{Introduction}

Acute myeloid leukemia (AML) is a hematologic malignancy, characterized by aberrant proliferation and differentiation of haematopoietic stem cells (HSCs) [1]. Compared to other types of leukemia, AML is more common in adults and its prevalence increases with age $[2,3]$.

Long non-coding RNAs (IncRNAs) belong to the non-coding RNAs family that are more than 200 nucleotides long [4]. LncRNAs have several molecular functions, such as regulating transcription in cis or trans, organizing nuclear domains, and regulating proteins or RNA molecules $[5,6]$. In recent years, several studies indicated that IncRNAs may play an important role in various cancers [7]. Meanwhile, there are also many IncRNAs, which could regulate the development and progression of AML. For example, Sun et al. revealed that the IncRNA ANRIL may promote malignant cell growth and glucose metabolism by regulating the ANRIL-AdipoR1-AMPK/SIRT1 signaling pathway [8]. Dong et al. showed that the IncRNA HOXA-AS2 regulates the chemoresistance of AML cells by competing for miR-520c-3p binding and through regulating S100A4 expression by acting as a ceRNA [9]. Shi et al. demonstrated that IncRNA LINP1 promotes AML progression and stimulates glucose metabolism by regulating the HNF4a/AMPK/WNT5A signaling pathway [10]. Furthermore, Feng et al. revealed that IncRNA NR-104098 suppresses EZH2 transcription by interacting with E2F1 to inhibit AML proliferation and promote its differentiation [11]. 
The Colorectal neoplasia differentially expressed (CRNDE) is a gene located on chromosome 16, that was first found to be overexpressed in colorectal adenomas and carcinomas, and that was confirmed to play a critical role in many types of cancers [12-14]. Moreover, its expression was shown to be upregulated in AML patients' bone marrow tissues and which negatively correlated with patients' overall survival [15]. However, CRNDE role in AML remains unexplored. In the present study, we demonstrate that CRNDE acts as a ceRNA by modulating AML progression via the CRNDE-miR-136-5p-MCM5 axis and provide a new regulatory network of CRNDE in AML.

\section{Materials And Methods}

\subsection{Cell culture}

The acute myeloid leukemia cell lines U937, KG-1a, NB4 and HL60, and the human embryonic kidney 293T cell line that were used for the experiments were purchased from American Type Culture Collection (ATCC, Manassas, VA, USA). RPMI-1640 medium (Gibco, USA), supplemented with 10\% fetal bovine serum (FBS, USA) and 1\% penicillin-streptomycin (Beyotime biotech, China) was used to culture the AML cells. The 293T cells were cultured in Dulbecco's Modified Eagle's Medium (DMEM, Gibco, USA), supplemented with $10 \%$ fetal bovine serum (FBS, USA) and 1\% penicillin-streptomycin (Beyotime biotech, China). The cells were maintained at $37^{\circ} \mathrm{C}$ with $5 \% \mathrm{CO}_{2}$. The leukocytes were obtained from healthy human peripheral blood.

\subsection{Reverse transcription $P C R$ and quantitative real-time $P C R$}

TRIZOL reagent (Takara, Japan) was used to extract total RNA, that was transcribed into cDNA using the PrimeScript $^{\text {TM }}$ RT reagent Kit (Takara, Japan). Quantitative real-time PCR (qRT-PCR) was performed on a CFX Connect ${ }^{\text {TM }}$ real-time PCR operating system (Bio-Rad, USA) using the SYBR ${ }^{\circledR}$ Premix Ex Taq ${ }^{\text {TM }}$ II (Takara, Japan) kit. The used primers are listed in Table 1. Relative expression levels were calculated using the $2^{-\Delta \Delta \mathrm{Ct}}$ method. $\mathrm{U} 6$ and $\beta$-actin were used as internal standards for miRNA and mRNA, respectively.

Table 1. Sequences used for RT-PCR and qRT-PCR 


\begin{tabular}{|ll|}
\hline Genes & Sequences $\left(\mathbf{5}^{\prime}\right.$-3') \\
\hline CRNDE & F: ATATTCAGCCGTTGGTCTTTGA \\
RiR-136-5p & F: : CGCTGCGTGACAACTGAGGATTT \\
& R: AGTGCAGGGTCCGAGGTATT \\
& RT: GTCGTATCCAGTGCAGGGTCCGAGGT \\
& ATTCGCACTGGATACGACTCCATC \\
MCM5 & F: AGCATTCGTAGCCTGAAGTCG \\
B-Actin & F: CGGCACTGGATAGAGATGCG \\
& R: CTGGAAGGTGGACAGCGAGG \\
\hline U6 & F: GCTTCGGCAGCACATATACTAAAAT \\
& R: CGCTTCACGAATTTGCGTGTCAT \\
\hline
\end{tabular}

\subsection{Cell transfection and infection}

MiR-136-5p mimic, miR-136-5p inhibitor, the mimic and the inhibitor negative controls were purchased from GenePharma (Shanghai, China). Briefly, $1 \times 10^{6}$ cells per well were seeded in a 6-well plate and transfected using Lipofectamine 2000 (Invitrogen, USA), according to the manufacturer's protocols.

To generate CRNDE silenced stable cell lines, we used a lentiviral vector, which was designed and synthesized by GenePharma (Shanghai, China). The U937 and KG-1a cells were transfected following the manufacturer's protocols, and after $72 \mathrm{~h}$ incubation, the cells were selected by adding $5 \mu \mathrm{g} / \mathrm{mL}$ puromycin (Abcam, UK) for approximatively 2 weeks to generate the stable cell lines.

\subsection{Cell proliferation assay}

Cell proliferation was detected using the Cell Counting Kit-8 (CCK8; HY-K0301), that was purchased from MedChemExpress (NJ, USA). The transfected cells were seeded into 96-well plates at a density of $2 \times 10^{3}$ /well, according to the manufacturer's protocols, and $10 \mu$ l of CCK-8 was added to each well at 0,12 , $24,36,48 \mathrm{~h}$, followed by an additional $2 \mathrm{~h}$ incubation. The OD value at $450 \mathrm{~nm}$ was measured using a microplate reader.

\subsection{Cell Counting}


The transfected cells were seeded into 6-well plates and cultured at $37^{\circ} \mathrm{C}$ with $5 \% \mathrm{CO}_{2}$ and the numbers of cells were counted at $0,24,48,72$ and $96 \mathrm{~h}$.

\subsection{Flow cytometry}

To assess cell apoptosis, the transfected cells were collected, washed twice with cold phosphate-buffered saline (PBS), centrifuged at $1000 \mathrm{rpm}$ for $5 \mathrm{~min}$ and resuspended at approximatively $1 \times 10^{6}$ cells in $500 \mu \mathrm{l}$ PBS. For cell cycle analysis, the cells were washed twice, resuspended in $100 \mu \mathrm{L}$ PBS and $500 \mu \mathrm{l}$ of prechilled $75 \%$ ethanol was slowly added prior an overnight storage at $4^{\circ} \mathrm{C}$. Apoptosis and cell cycle were assessed by CytoFLEX flow cytometry (Beckman, USA), and the Cytexpert and Kaluza Analysis software were respectively used for data acquisition and analysis.

\subsection{Western blot}

The cells were collected, washed 3 times with cold PBS and treated with cold RIPA lysis buffer containing a protease inhibitor cocktail (Beyotime Biotechnology, Shanghai, China). The BCA protein assay kit was used to detect the protein concentration, according to the manufacturer's protocol (Beyotime Biotechnology). The extracted proteins were separated on SDS-PAGE gels and transferred to PVDF membranes (Millipore, MA, USA). The membranes were blocked with $5 \%$ skim milk for $2 \mathrm{~h}$ at room temperature and then the following primary antibodies were added: $\beta$-Actin (BOSTER Biological Technology, CA, USA), MCM5 (Abcam, MA, USA), Bcl-2 (Abcam, MA, USA), CyclinD1 (Wanleibio, Shenyang, China), CyclinA2 (Wanleibio, Shenyang, China) and p53 (Wanleibio, Shenyang, China). This step was followed by the addition of horseradish peroxidase-conjugated secondary antibodies $(1: 4,000$; Biosharp, China) and the signals were detected using an ECL (enhanced chemiluminescence) kit (Millipore, USA).

\subsection{Luciferase reporter assays}

The luciferase reporter plasmids CRNDE-Wt, CRNDE-Mut, MCM5-Wt and MCM5-Mut were purchased from GeneCreate (Wuhan, China). The sequences were respectively cloned into a pmirGLO Dual-luciferase plasmid to construct the luciferase reporter vectors. Lipofectamine 2000 (Invitrogen, CA, USA) was used to co-transfect the luciferase reporter plasmids and miR-136-5p mimic or mimic negative control into 293T cells. After $48 \mathrm{~h}$ culture, the transfected cells were collected and the luciferase activity measured using the Dual-Luciferase Reporter Assay System kit (Promega, WI, USA). The Firefly luciferase activity was standardized by the Renilla luciferase.

\subsection{RNA immunoprecipitation (RIP) assay}

An RNA Binding Protein Immunoprecipitation kit (Millipore, Burlington, MA) was used to determine whether CRNDE exists in the miRNA mediated RISC complex. The cells were lysed in lysis buffer and antiAgo2 antibody or anti-IgG magnetic beads were used to immunoprecipitate target RNA binding proteins 
and then the precipitated RNA was separated. QPCR was performed to analyze the expression of Linc00210.

\subsection{Statistical Analysis}

Each experiment included at least 3 independent replicates and all values were shown as means \pm SD. All statistics were analyzed using GraphPad Prism 6. The comparison between the groups was analyzed using the Student's t-test or the one-way analysis of variance (ANOVA). A p-value $<0.05$ was considered significant $(*)$ and a $p$-value $<0.01$ was considered as highly significant $(* *)$.

\section{Results}

\subsection{CRNDE is highly expressed in AML}

To investigate the expression of IncRNA CRNDE in AML, we analyzed its expression data in The Cancer Genome Atlas (TCGA) database by GEPIA. The results showed that CRNDE mRNA expression was significantly upregulated in AML samples $(n=173)$, compared with that in normal samples $(n=70)$ (Figure 1a, 1b). Additionally, CRNDE high expression correlated with AML poor overall survival (OS) (Figures 1c). Furthermore, we examined the CRNDE expression in AML cell lines by qPCR and the results showed that CRNDE was significantly overexpressed in AML cell lines (U937, KG-1a, NB4 and HL60) compared to that in leukocytes (Figure 1d). These data indicated that CRNDE is highly expressed in AML.

\subsection{CRNDE knockdown inhibits proliferation and promotes apoptosis in AML cells}

To further explore the effects of CRNDE in AML, we transfected a specific CRNDE small hairpin RNA (shRNA) into U937 and KG-1a cells to knock down CRNDE and which efficiency was assessed by qPCR (Figure 2a). The cell counting kit-8 (CCK-8) and cell counting assay showed a significant inhibition of cell proliferation following CRNDE knockdown and in both cell lines (Figure 2b, 2c). Additionally, flow cytometry assays indicated that apoptosis was increased, and that the U937 and KG-1a cells were arrested at the $\mathrm{G} 1$ phase of the cell cycle (Figure 2d, 2f). These results were confirmed by Western blotting (Figure 2e, 2g). In conclusion, CRNDE knockdown inhibits proliferation, promotes apoptosis, and blocks the cell cycle at $\mathrm{G} 1$ phase in AML cells.

\subsection{CRNDE targets miR-136-5p and negatively regulates its expression}

The subcellular localization of IncRNA is closely related to its potential mechanism. LncLocator [16] predicted that CRNDE was mainly located in the cytosol, and that CRNDE most likely regulates AML as a competing endogenous RNA (ceRNA) (Figure 3a). Next, the prediction program Starbase V2.0 was used to determine its targets and we found the presence of a putative interaction site between CRNDE and miR136-5p (Figure 3b). When CRNDE was knocked down, miR-136-5p expression was significantly enhanced in U937 and KG-1a cells (Figure 3c). Additionally, miR-136-5p specific mimics and inhibitors were transfected into U937 and KG-1a cells and qPCR was used to detect the transfection efficiency (Figure $3 d)$. The results indicated that miR-136-5p inhibited CRNDE expression, while miR-136-5p downregulation 
rescued its expression (Figure 3e). Then, a luciferase reporter assay was performed according to the interaction site and its mutation. The results indicated that miR-136-5p overexpression significantly reduces the luciferase activity of the CRNDE-WT (Figure 3f). Furthermore, RIP assay indicated that CRNDE and miR-136-5p were highly enriched in the anti-Ago2 group compared to IgG control group (Figure $3 g$ ). Taken together, these results show that CRNDE binds to miR-136-5p in AML.

\subsection{MiR-136-5p is downregulated in AML cells and acts as tumor suppressor}

QPCR showed that miR-136-5P was significantly downregulated in AML cells compared to leukocytes (Figure 4a). The CCK-8 assay showed that cell proliferation was significantly inhibited by miR-136-5p overexpression, while miR-136-5p downregulation promoted proliferation in U937 and KG-1a cells (Figure 4b). Furthermore, flow cytometry assays were performed to assess the apoptotic rate and cell cycle. The overexpression of miR-136-5P increased the apoptotic rate, whereas its downregulation decreased this rate (Figure $4 \mathrm{c}$ ). The results also showed that the cells were arrested at $\mathrm{G} 1$ phase when miR-136-5P was overexpressed (Figure 4d). These results demonstrate that miR-136-5P acts as a tumor suppressor.

\subsection{MCM5 is a direct target of miR-136-5p}

To predict miR-136-5p direct target, TargetScan was used and the results showed that MCM5 was a putative target of miR-136-5p. The binding sites between miR-136-5p and MCM5 are as shown in the figure 5a. The qPCR results indicated that miR-136-5p inhibits MCM5 mRNA expression, while its expression increased following miR-136-5p downregulation (Figure $5 b$ ). These results were confirmed by Western blotting (Figure 5c). Furthermore, the luciferase reporter assay indicated that miR-136-5p overexpression directly inhibits MCM5 activity (Figure $5 \mathrm{~d}$ ).

\subsection{CRNDE regulates MCM5 through sponging miR-136-5p}

To explore the effect of MCM5 in AML, we analyzed MCM5 expression data by GEPIA. The results revealed that high MCM5 expression correlates with poor overall survival in AML patients (Figure 6a). Additionally, we also investigated the MCM5 expression in AML cell lines by $\mathrm{qPCR}$, and the results showed that its expression was high expressed in AML cell lines (U937, KG-1a, NB4 and HL60) compared to that in leukocytes (Figure 6b). Moreover, the qPCR and Western blot results indicated that CRNDE knockdown inhibits MCM5 expression at the mRNA and protein levels (Figure 6c, d). Rescue assays revealed that these effects could be reversed by miR-136-5p inhibitors, at both mRNA and protein levels (Figure 6e, f), which further showed that CRNDE regulates MCM5 through sponging miR-136-5p. The possible modulating mechanism of CRNDE in AML cells through miR-136-5p sponging is shown in figure 7.

\section{Discussion}

Although a few chemotherapeutic drugs were applied in AML treatment, aged AML patients still suffer from poor prognosis and low long-term overall survival rate [17]. AML occurrence and development are 
affected by multiple factors, such as radiation, chemical degradation, viral infection, and genes' susceptibility [18]. In recent years, increasing evidence revealed that IncRNAs play vital biological functions in tumorigenesis $[19,20]$. For example, Fang et al. found that XIST promotes cell proliferation, migration, and invasion through epigenetically suppressing the KLF2 family of proteins [21]. Chen et al. revealed that CCAT1 acts as a molecular sponge of miR-181a-5p, regulates HOXA1 expression and promotes multiple myeloma progression [22]. Therefore, it is imperative to explore the potential mechanism of IncRNAs in hematological malignancies.

Emerging evidence showed that CRNDE regulates cancer progression [23-26]. For example, Zheng et al. confirmed that CRNDE is the most upregulated IncRNA in glioma, where it promotes its progression by inhibiting the miR-384/PIWIL4/STAT3 axis [27]. Xu et al. revealed that CRNDE promotes melanoma migration and invasion via the miR-205/CCL18 axis [28]. CRNDE expression has been shown to be upregulated in the bone marrow tissues of AML patients and negatively correlated patients' overall survival [15]. However, the underlying mechanisms of CRNDE in AML remain unexplored. In this study, we confirmed that the expression of CRNDE was significantly upregulated in AML samples and cell lines. CRNDE promoted AML cells' proliferation ability, decreases AML cells' apoptotic rate and arrested AML cells at the $\mathrm{G} 1$ phase.

The hypothesis of ceRNA was first presented by Salmena et al., and explained how messenger RNAs, transcribed pseudogenes, and long non-coding RNAs communicate with each other using microRNA response elements (MREs). They proposed that the "competing endogenous RNA" (ceRNA) could bind and negatively regulate the target [29]. Moreover, the function of IncRNAs was found to be closely related to its subcellular location [30]. In this study, the IncLocator that predicts IncRNAs subcellular localizations was used to predict the location of CRNDE [16]. We predicted that CRNDE was mainly located in the cytosol where it potentially acts as a ceRNA. Indeed, functional, and mechanistic experiments showed that CRNDE serves as a ceRNA for miR-136-5p and that it upregulates MCM5 expression by sponging miR-136-5p.

MiRNAs are a class of small single-stranded RNAs with a length of 18 to 25 nucleotides. Moreover, miRNAs were confirmed to be involved in cell growth, cell apoptosis, hematopoietic lineage differentiation and cell death [31-33]. Chen et al. revealed that miR-136-5p could inhibit cell growth, migration, and invasion of renal cell carcinoma cells [34]. Gao et al. demonstrated that miR-136-5p may play an important role in regulating TC tumorigenesis via targeting MTDH [35]. In the present study, the results of the bioinformatics software Starbase V2.0 showed that binding sites exist between CRNDE and miR-136$5 p$, which was proved by luciferase reporter assays. In addition, rescue assays also showed that CRNDE indeed influences MCM5 via acting on miR-136-5p.

Subsequently, we further explored the downstream target genes using the bioinformatics' software Targetscan 7.0 and filtered out MCM5 as a miR-136-5p direct target. Minichromosome maintenance protein 5 (MCM5) is a member of the minichromosome maintenance (MCM) protein family, that is located on chromosome 22q13.1 [36]. MCM proteins were first identified in the yeast Saccharomyces 
cerevisiae as mutants defective in the maintenance of minichromosomes, indicating their role in plasmid replication and cell cycle progression [37]. MCM5 was well documented to play a role in tumorigenesis and cellular processes in various cancers. For example, Gong et al. revealed that MCM5 could be related to the prognosis and progression of renal cell carcinoma [38]. Guida et al. found that targeting MCM proteins could be a used as a therapy for anaplastic thyroid carcinomas by disturbing their expression or obstructing their helicase function [39]. It has also been proven that MCM5 had could be used a prognostic marker in various cancers [40-45]. In addition, MCM5 was confirmed to be overexpressed in AML by boutique microarrays, real-time PCR, and droplet digital PCR [46]. In our study, we also verified that MCM5 was upregulated in AML and associated with poor prognostic. Luciferase reporter assays showed that MCM5 was a downstream target of miR-136-5p, and that MCM5 expression was downregulated when CRNDE was knocked down or when miR-136-5p was overexpressed.

In the present study, we demonstrated the potential mechanistic role of CRNDE in AML; however, there are still some limitations associated with the study. Firstly, we did not elucidate the cause of CRNDE upregulation in AML, which is necessary for a better understanding of CRNDE mechanism of action. Secondly, our research was performed at the cellular level and it is therefore, important to study its effect in vivo and the future.

\section{Conclusion}

In summary, we expounded that CRNDE plays a pro-oncogenic role in AML, where it promoted proliferation by modulating the miR-136-5p/MCM5 axis. CRNDE-targeted therapy may be an effective strategy for the treatment of $A M L$ patients.

\section{Abbreviations}

AML: Acute myeloid leukemia; CRNDE: colorectal neoplasia differentially expressed; MCM5: minichromosome maintenance protein 5; IncRNA: long non-coding RNA; ceRNA: Competitive endogenous RNA; miRNAs: MicroRNAs.

\section{Declarations}

\section{Acknowledgements}

Not applicable.

\section{Authors' contributions}

$\mathrm{CL}$ as the principal investigator was responsible for the concept, data curation, formal analysis, funding acquisition, investigation, methodology and writing-original draft. LZ was responsible for conceptualization, project administration, resources and supervision. CS, XC, XL, LY, JY, LX, WD, JL did the investigation and methodology. BL was responsible for conceptualization, funding acquisition, project 
administration, resources, supervision, writing-review and editing. All authors read and approved the final manuscript.

\section{Funding}

This work was supported by grants from the National Natural Science Foundation of China (Grant Number 81772280) and the Open Research Fund of the Key Laboratory of Biotherapy, West China Hospital, West China Medicine School, Sichuan University (Grant Number SKLB202008).

\section{Availability of data and materials}

All data analyzed and generated during the current study are available from the corresponding author upon reasonable request.

\section{Ethics approval and consent to participate}

Not applicable, no human subjects.

\section{Consent for publication}

Not applicable, no human subjects.

\section{Competing interests}

The authors declare that that they have no competing interests.

\section{Author details}

1 Central Laboratory of Yong-Chuan Hospital, Chongqing Medical University, Chongqing 402160, China. 2 Key Laboratory of Laboratory Medical Diagnostics, Ministry of Education, Department of Laboratory Medicine, Chongqing Medical University, Chongqing 400016, China. 3 Clinical Laboratory of Yong-Chuan Hospital, Chongqing Medical University, Chongqing 402160, China.

\section{References}

1. Short NJ, Rytting ME, Cortes JE: Acute myeloid leukaemia. Lancet (London, England) 2018, 392(10147):593-606.

2. Khwaja A, Bjorkholm M, Gale RE, Levine RL, Jordan CT, Ehninger G, Bloomfield CD, Estey E, Burnett A, Cornelissen JJ et al: Acute myeloid leukaemia. Nature reviews Disease primers 2016, 2:16010.

3. Meyers J, Yu Y, Kaye JA, Davis KL: Medicare fee-for-service enrollees with primary acute myeloid leukemia: an analysis of treatment patterns, survival, and healthcare resource utilization and costs. Applied health economics and health policy 2013, 11(3):275-286.

4. Ponting CP, Oliver PL, Reik W: Evolution and functions of long noncoding RNAs. Cel/ 2009, 136(4):629-641. 
5. Wang KC, Chang HY: Molecular mechanisms of long noncoding RNAs. Molecular cell 2011, 43(6):904-914.

6. Kopp F, Mendell JT: Functional Classification and Experimental Dissection of Long Noncoding RNAs. Cell 2018, 172(3):393-407.

7. Schmitt AM, Chang HY: Long Noncoding RNAs in Cancer Pathways. Cancer cell 2016, 29(4):452-463.

8. Sun LY, Li XJ, Sun YM, Huang W, Fang K, Han C, Chen ZH, Luo XQ, Chen YQ, Wang WT: LncRNA ANRIL regulates AML development through modulating the glucose metabolism pathway of AdipoR1/AMPK/SIRT1. Molecular cancer 2018, 17(1):127.

9. Dong X, Fang Z, Yu M, Zhang L, Xiao R, Li X, Pan G, Liu J: Knockdown of Long Noncoding RNA HOXA-AS2 Suppresses Chemoresistance of Acute Myeloid Leukemia via the miR-520c-3p/S100A4 Axis. Cellular physiology and biochemistry : international journal of experimental cellular physiology, biochemistry, and pharmacology 2018, 51(2):886-896.

10. Shi J, Dai R, Chen Y, Guo H, Han Y, Zhang Y: LncRNA LINP1 regulates acute myeloid leukemia progression via HNF4a/AMPK/WNT5A signaling pathway. Hematological oncology 2019, 37(4):474482.

11. Feng Y, Hu S, Li L, Zhang S, Liu J, Xu X, Zhang M, Du T, Du Y, Peng X et al: LncRNA NR-104098 Inhibits AML Proliferation and Induces Differentiation Through Repressing EZH2 Transcription by Interacting With E2F1. Frontiers in cell and developmental biology 2020, 8:142.

12. Graham LD, Pedersen SK, Brown GS, Ho T, Kassir Z, Moynihan AT, Vizgoft EK, Dunne R, Pimlott L, Young GP et al: Colorectal Neoplasia Differentially Expressed (CRNDE), a Novel Gene with Elevated Expression in Colorectal Adenomas and Adenocarcinomas. Genes \& cancer 2011, 2(8):829-840.

13. Ellis BC, Molloy PL, Graham LD: CRNDE: A Long Non-Coding RNA Involved in CanceR, Neurobiology, and DEvelopment. Frontiers in genetics 2012, 3:270.

14. Zhang J, Yin M, Peng G, Zhao Y: CRNDE: An important oncogenic long non-coding RNA in human cancers. Cell proliferation 2018, 51(3):e12440.

15. Wang Y, Zhou Q, Ma JJ: High expression of Inc-CRNDE presents as a biomarker for acute myeloid leukemia and promotes the malignant progression in acute myeloid leukemia cell line U937. European review for medical and pharmacological sciences 2018, 22(3):763-770.

16. Cao Z, Pan X, Yang Y, Huang Y, Shen HB: The IncLocator: a subcellular localization predictor for long non-coding RNAs based on a stacked ensemble classifier. Bioinformatics (Oxford, England) 2018, 34(13):2185-2194.

17. So CC, Wan TS, Chow JL, Hui KC, Choi WW, Lam CC, Chan LC: A single-center cytogenetic study of 629 Chinese patients with de novo acute myeloid leukemia-evidence of major ethnic differences and a high prevalence of acute promyelocytic leukemia in Chinese patients. Cancer genetics 2011, 204(8):430-438.

18. Carey A, Edwards DKt, Eide CA, Newell L, Traer E, Medeiros BC, Pollyea DA, Deininger MW, Collins RH, Tyner JW et al: Identification of Interleukin-1 by Functional Screening as a Key Mediator of Cellular 
Expansion and Disease Progression in Acute Myeloid Leukemia. Cell reports 2017, 18(13):32043218.

19. Mercer TR, Dinger ME, Mattick JS: Long non-coding RNAs: insights into functions. Nature reviews Genetics 2009, 10(3):155-159.

20. Quinn JJ, Chang HY: Unique features of long non-coding RNA biogenesis and function. Nature reviews Genetics 2016, 17(1):47-62.

21. Fang J, Sun CC, Gong C: Long noncoding RNA XIST acts as an oncogene in non-small cell lung cancer by epigenetically repressing KLF2 expression. Biochemical and biophysical research communications 2016, 478(2):811-817.

22. Chen L, Hu N, Wang C, Zhao H, Gu Y: Long non-coding RNA CCAT1 promotes multiple myeloma progression by acting as a molecular sponge of miR-181a-5p to modulate HOXA1 expression. Cell cycle (Georgetown, Tex) 2018, 17(3):319-329.

23. Chen Z, Yu C, Zhan L, Pan Y, Chen L, Sun C: LncRNA CRNDE promotes hepatic carcinoma cell proliferation, migration and invasion by suppressing miR-384. American journal of cancer research 2016, 6(10):2299-2309.

24. Li DX, Fei XR, Dong YF, Cheng CD, Yang Y, Deng XF, Huang HL, Niu WX, Zhou CX, Xia CY et al: The long non-coding RNA CRNDE acts as a ceRNA and promotes glioma malignancy by preventing miR136-5p-mediated downregulation of Bcl-2 and Wnt2. Oncotarget 2017, 8(50):88163-88178.

25. Tang Q, Zheng X, Zhang J: Long non-coding RNA CRNDE promotes heptaocellular carcinoma cell proliferation by regulating PI3K/Akt / $\beta$-catenin signaling. Biomedicine \& pharmacotherapy $=$ Biomedecine \& pharmacotherapie 2018, 103:1187-1193.

26. Zhang M, Gao C, Yang Y, Li G, Dong J, Ai Y, Chen N, Li W: Long Noncoding RNA CRNDE/PRC2 Participated in the Radiotherapy Resistance of Human Lung Adenocarcinoma Through Targeting p21 Expression. Oncology research 2018, 26(8):1245-1255.

27. Zheng J, Liu X, Wang P, Xue Y, Ma J, Qu C, Liu Y: CRNDE Promotes Malignant Progression of Glioma by Attenuating miR-384/PIWIL4/STAT3 Axis. Molecular therapy : the journal of the American Society of Gene Therapy 2016, 24(7):1199-1215.

28. Xu L, Zhang Y, Zhao Z, Chen Z, Wang Z, Xu S, Zhang X, Liu T, Yu S: The long non-coding RNA CRNDE competed endogenously with miR-205 to promote proliferation and metastasis of melanoma cells by targeting CCL18. Cell cycle (Georgetown, Tex) 2018, 17(18):2296-2308.

29. Salmena L, Poliseno L, Tay Y, Kats L, Pandolfi PP: A ceRNA hypothesis: the Rosetta Stone of a hidden RNA language? Cell 2011, 146(3):353-358.

30. Chen LL: Linking Long Noncoding RNA Localization and Function. Trends in biochemical sciences 2016, 41(9):761-772.

31. Cheng AM, Byrom MW, Shelton J, Ford LP: Antisense inhibition of human miRNAs and indications for an involvement of miRNA in cell growth and apoptosis. Nucleic acids research 2005, 33(4):12901297. 
32. Chen CZ, Li L, Lodish HF, Bartel DP: MicroRNAs modulate hematopoietic lineage differentiation. Science (New York, NY) 2004, 303(5654):83-86.

33. Bartel DP: MicroRNAs: genomics, biogenesis, mechanism, and function. Cel/ 2004, 116(2):281-297.

34. Chen P, Zhao L, Pan X, Jin L, Lin C, Xu W, Xu J, Guan X, Wu X, Wang Y et al: Tumor suppressor microRNA-136-5p regulates the cellular function of renal cell carcinoma. Oncology letters 2018, 15(4):5995-6002.

35. Gao RZ, Que Q, Lin P, Pang YY, Wu HY, Li XJ, Chen G, He Y, Yang H: Clinical roles of miR-136-5p and its target metadherin in thyroid carcinoma. American journal of translational research 2019, 11(11):6754-6774.

36. Yu SY, Wang YP, Chang JY, Shen WR, Chen HM, Chiang CP: Increased expression of MCM5 is significantly associated with aggressive progression and poor prognosis of oral squamous cell carcinoma. Journal of oral pathology \& medicine : official publication of the International Association of Oral Pathologists and the American Academy of Oral Pathology 2014, 43(5):344-349.

37. Tye BK: MCM proteins in DNA replication. Annual review of biochemistry 1999, 68:649-686.

38. Gong B, Ma M, Yang X, Xie W, Luo Y, Sun T: MCM5 promotes tumour proliferation and correlates with the progression and prognosis of renal cell carcinoma. International urology and nephrology 2019, 51(9):1517-1526.

39. Guida T, Salvatore G, Faviana P, Giannini R, Garcia-Rostan G, Provitera L, Basolo F, Fusco A, Carlomagno F, Santoro M: Mitogenic effects of the up-regulation of minichromosome maintenance proteins in anaplastic thyroid carcinoma. The Journal of clinical endocrinology and metabolism 2005, 90(8):4703-4709.

40. Korkolopoulou P, Givalos N, Saetta A, Goudopoulou A, Gakiopoulou H, Thymara I, Thomas-Tsagli E, Patsouris E: Minichromosome maintenance proteins 2 and 5 expression in muscle-invasive urothelial cancer: a multivariate survival study including proliferation markers and cell cycle regulators. Human pathology 2005, 36(8):899-907.

41. Gakiopoulou H, Korkolopoulou P, Levidou G, Thymara I, Saetta A, Piperi C, Givalos N, Vassilopoulos I, Ventouri $\mathrm{K}$, Tsenga A et al: Minichromosome maintenance proteins 2 and $\mathbf{5}$ in non-benign epithelial ovarian tumours: relationship with cell cycle regulators and prognostic implications. British journal of cancer 2007, 97(8):1124-1134.

42. Giaginis C, Georgiadou M, Dimakopoulou K, Tsourouflis G, Gatzidou E, Kouraklis G, Theocharis S: Clinical significance of MCM-2 and MCM- 5 expression in colon cancer: association with clinicopathological parameters and tumor proliferative capacity. Digestive diseases and sciences 2009, 54(2):282-291.

43. Gou K, Liu J, Feng X, Li H, Yuan Y, Xing C: Expression of Minichromosome Maintenance Proteins (MCM) and Cancer Prognosis: A meta-analysis. Journal of Cancer 2018, 9(8):1518-1526.

44. Liu YZ, Wang BS, Jiang YY, Cao J, Hao JJ, Zhang Y, Xu X, Cai Y, Wang MR: MCMs expression in lung cancer: implication of prognostic significance. Journal of Cancer 2017, 8(18):3641-3647. 
45. Wang D, Li Q, Li Y, Wang H: The role of MCM5 expression in cervical cancer: Correlation with progression and prognosis. Biomedicine \& pharmacotherapy $=$ Biomedecine \& pharmacotherapie 2018, 98:165-172.

46. Handschuh L, Kaźmierczak M, Milewski MC, Góralski M, Łuczak M, Wojtaszewska M, UszczyńskaRatajczak B, Lewandowski K, Komarnicki M, Figlerowicz M: Gene expression profiling of acute myeloid leukemia samples from adult patients with AML-M1 and $-\mathrm{M} 2$ through boutique microarrays, real-time PCR and droplet digital PCR. International journal of oncology 2018, 52(3):656-678.

\section{Figures}

a

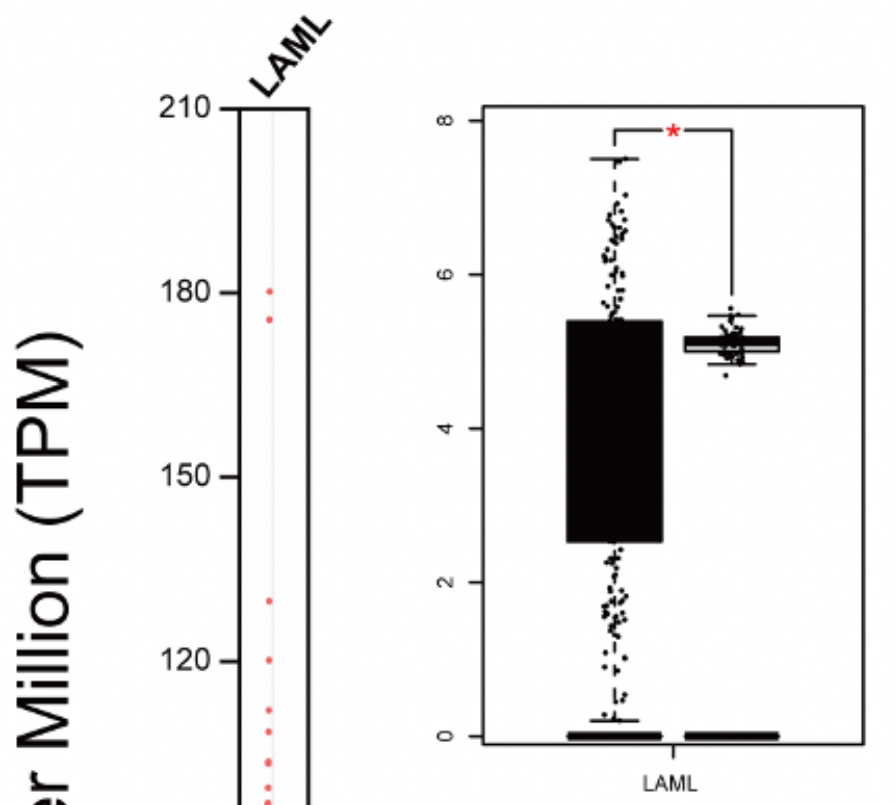

C

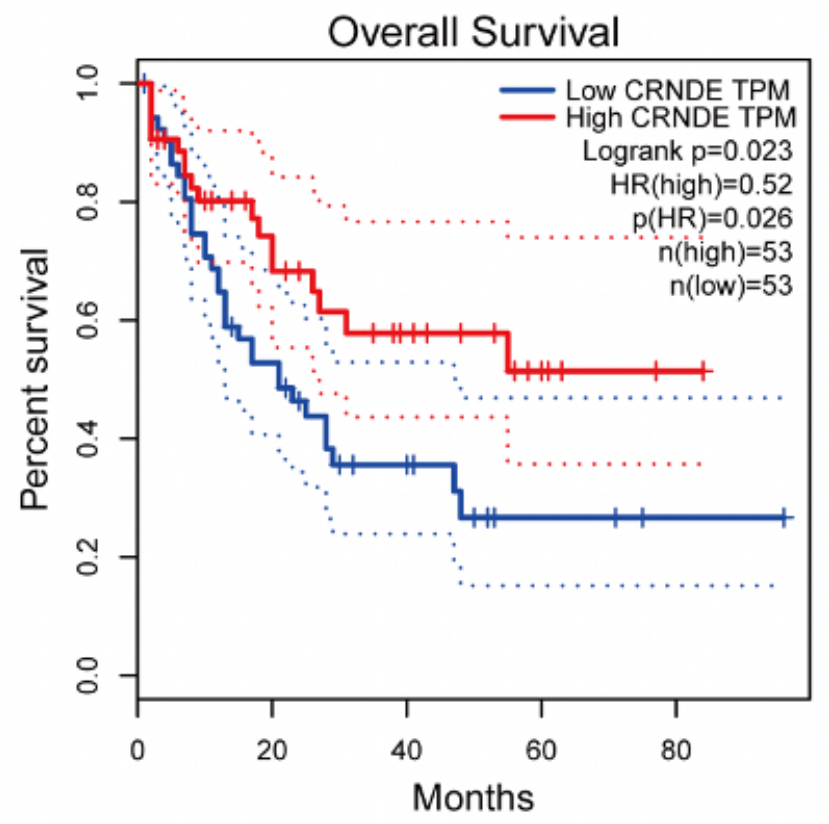

d

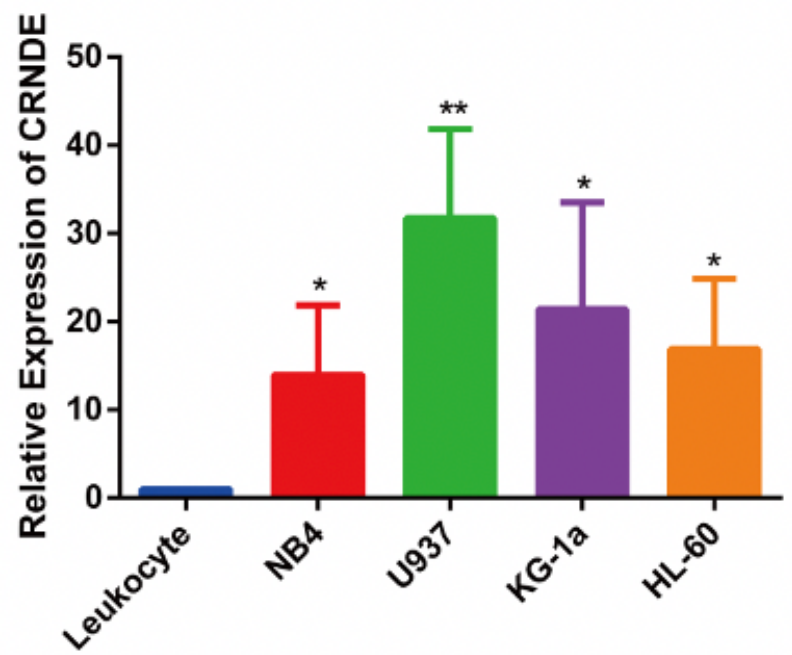

Figure 1 
High expression of CRNDE in AML. a-b The expression of CRNDE in normal samples $(n=70)$ and AML samples $(n=173)$ from GEPIA analysis. $c$ The overall survival curves of CRNDE in AML patients from GEPIA analysis. d CRNDE expression in AML cell lines (U937 and KG-1a) was detected by QPCR. *p < $0.05, * * p<0.01$

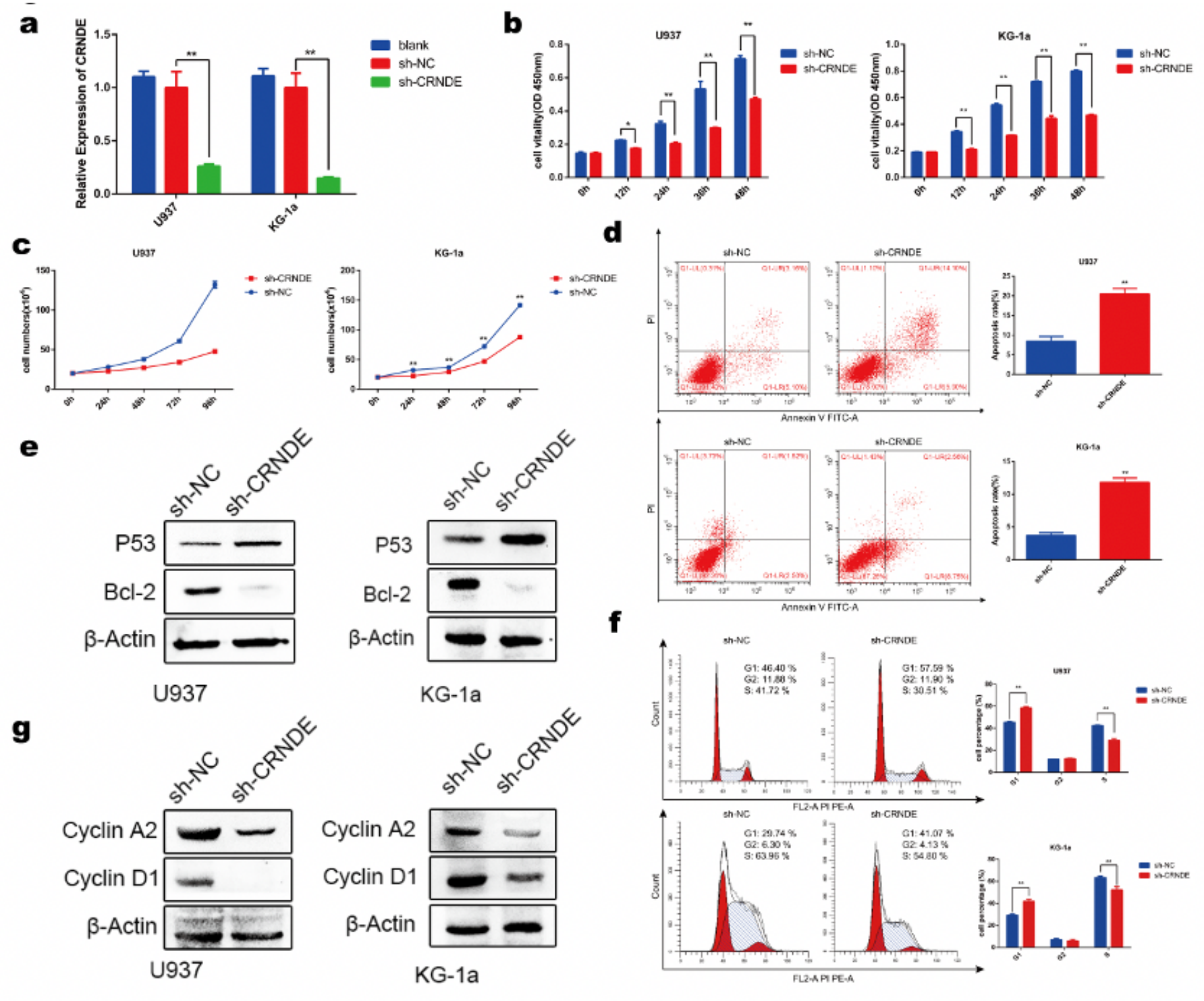

\section{Figure 2}

The effects of CRNDE knockdown on U937 and KG-1a cells proliferation, apoptosis and cell cycle distribution. a The transfection efficiency was verified by QPCR. b-c Cell viability was analyzed by the CCK-8 assay and cell counting assay. d-e Cell apoptosis was detected by flow cytometry and western blot. f-g Cell cycle was detected by flow cytometry and western blot. ${ }^{\star} p<0.05,{ }^{\star \star} p<0.01$ 


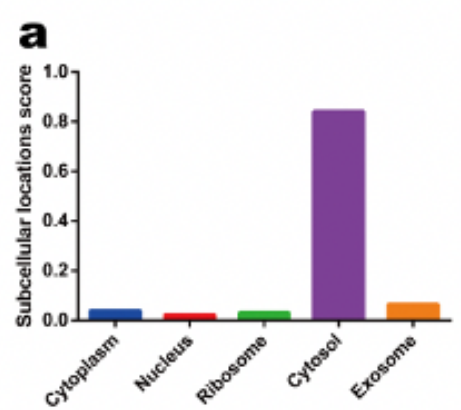

d

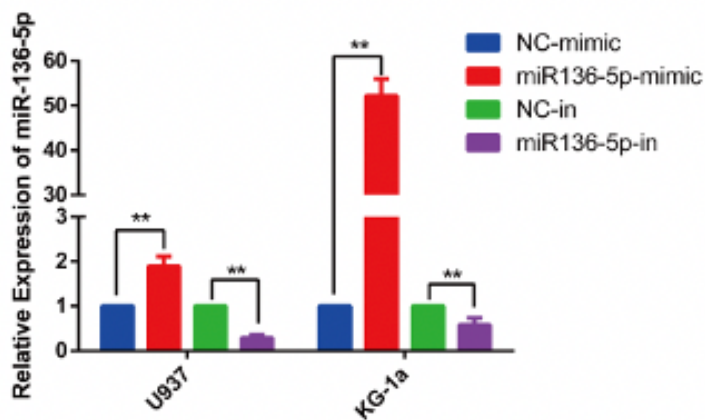

f

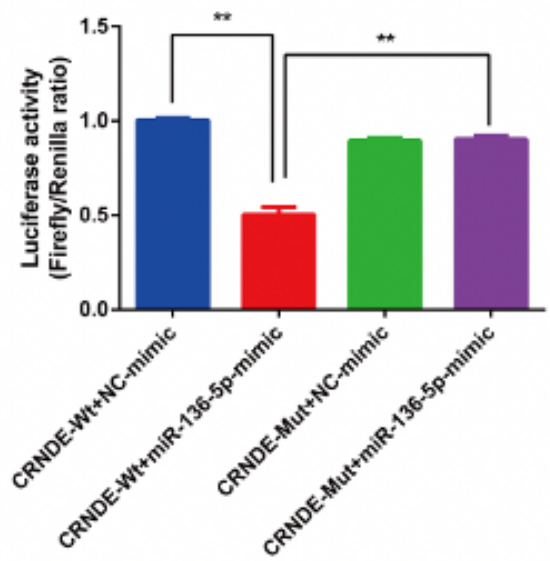

b

hsa-miR-136-5p 3'-AGGUAGUAGUUUUGUUUACCUCA-5'

InCRNA CRNDE 5'-CUAGGAAGAAAAUCAAAUGGAGU-3'

\section{$5^{\prime}$ Luciferase}

$\downarrow$

InCRNA CRNDE-Wt 5'-CUAGGAAGAAAAUCAAAUGGAGU-3' InCRNA CRNDE-Mut 5'-CUAGGAAGAUUUUGUUUACCUCU-3'
C

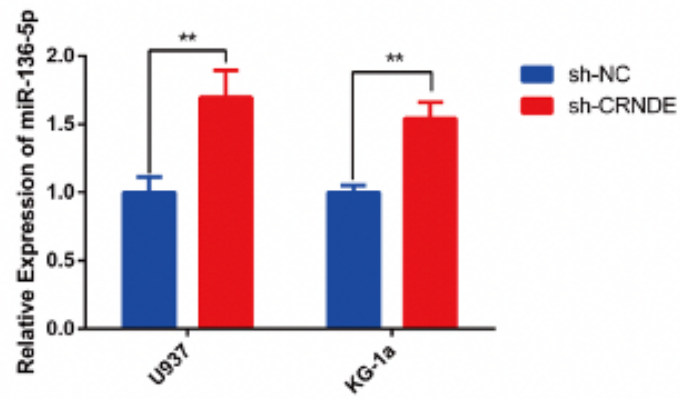

e

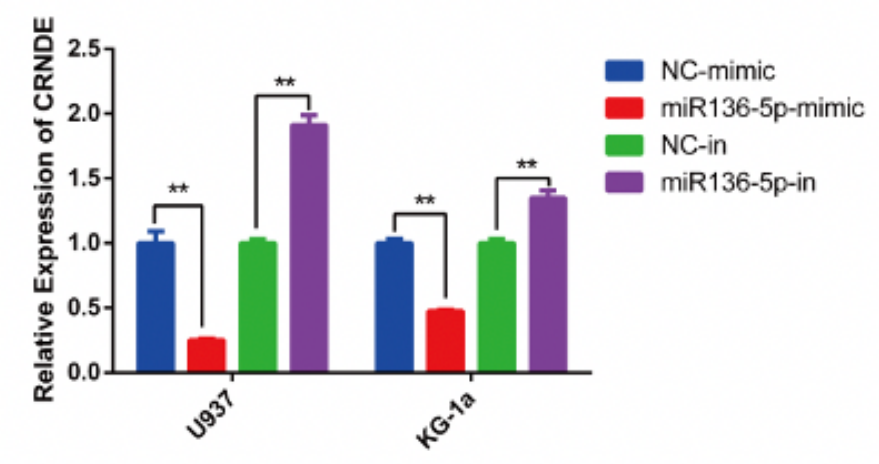

g

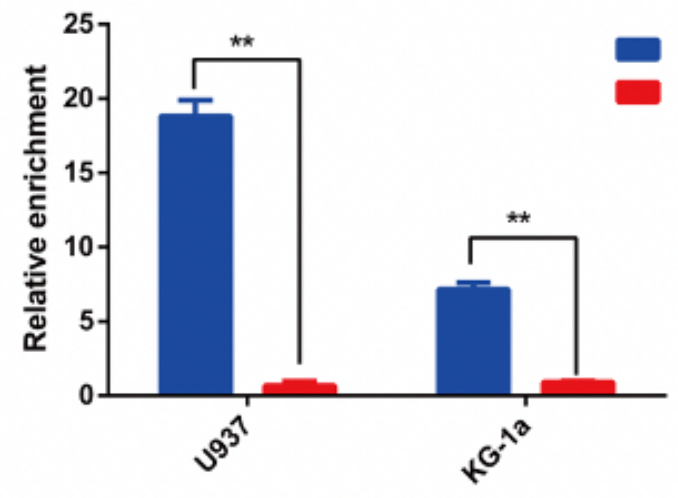

Ago2

lgG

Figure 3

CRNDE binds to miR-136-5p. a The subcellular localization of CRNDE was predicted by IncLocater. $b$ The binding sites between CRNDE and miR-136-5p were predicted by Starbase V2.0. c QPCR assay was used to detect the expression of miR-136-5p after knocking down CRNDE. d QPCR assay was used to detect the transfection efficiency of miR-136-5p mimic and inhibitor. e QPCR assay was used to determine the expression of CRNDE after upregulating or downregulating miR-136-5p. $f$ Luciferase reporter analysis of miR-382-5p mimics/ mimics-NC co-transfected with either CRNDE-wt or CRNDE-mut vector into 293T cells. g RIP assay was used to detect CRNDE expression in Ago2 immunoprecipitates. ${ }^{\star} p<0.05$, ${ }^{\star \star} p<$ 0.01 

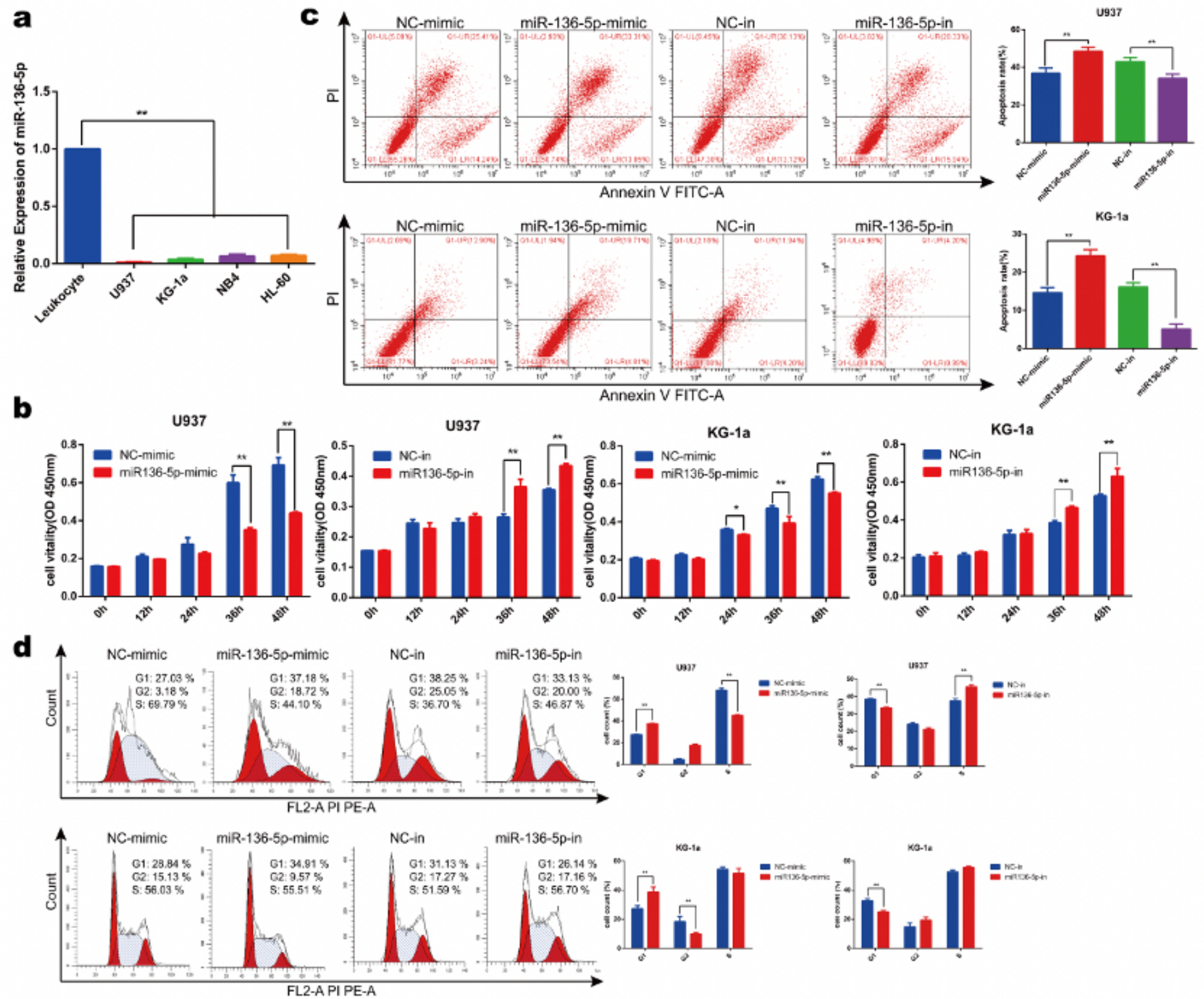

Figure 4

The effects of miR-136-5p on U937 and KG-1a cells proliferation, apoptosis and cell cycle distribution. a QPCR was applied to determine the expression of miR-136-5P in AML cells. b Cell viability was analyzed by CCK-8 assay. c Flow cytometry assay was performed to detect the apoptosis rate. $d$ Flow cytometry assay was performed to detect cell cycle distribution. ${ }^{*} p<0.05,{ }^{\star \star} p<0.01$ 
a

hsa-miR-136-5p 3'-AGGUAGUAGUUUUGUUUACCUCA-5' MCM5 3'UTR 5'-CAUACUGUUCGGGGAAAUGGAGA-3'

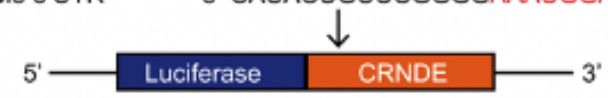

MCM5 3'UTR-Wt 5'-CAUACUGUUCGgGGAAAUGGAGA-3' MCM5 3'UTR-Mut 5'-CAUACUGUUCGgGGUUUACCUCA-3'

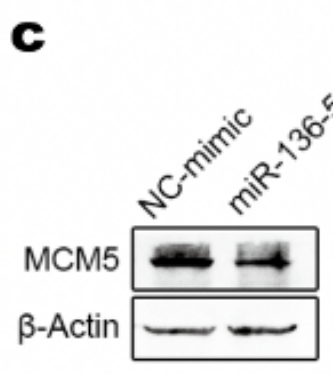

U937

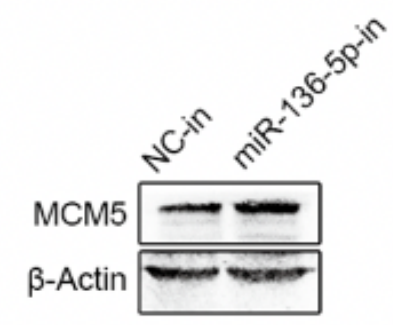

U937

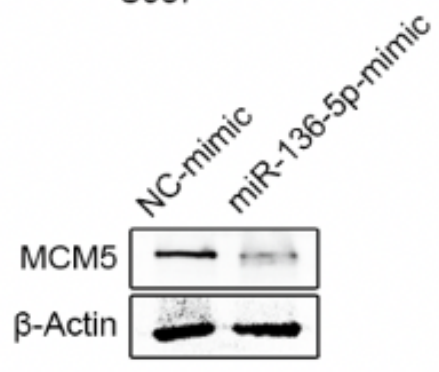

KG-1a

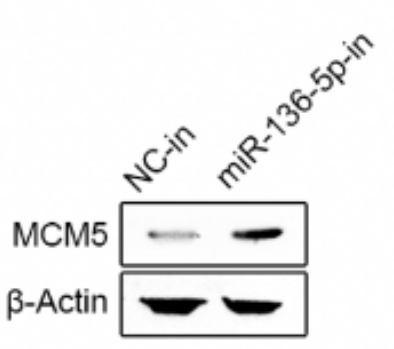

KG-1a

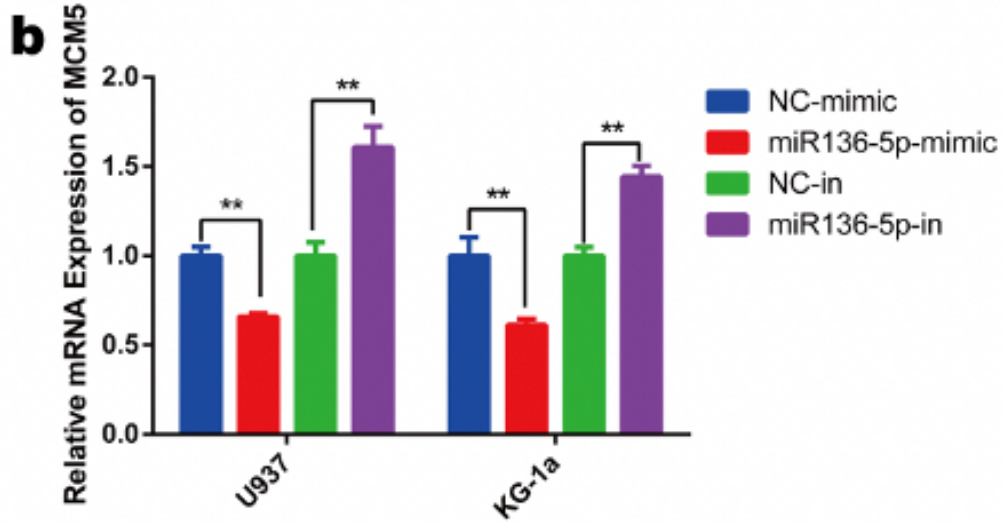

d

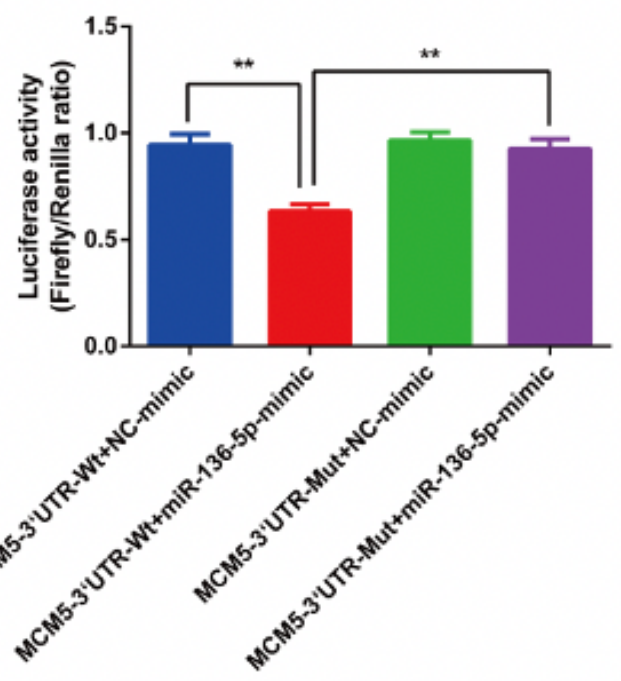

\section{Figure 5}

MiR-136-5p targets MCM5. a The binding sites between MCM5 and miR-136-5p were predicted by TargetScan. b QPCR assay was used to detect the expression of MCM5 mRNA after transfecting mimic or inhibitor. c The expression of MCM5 protein was detected by western blot. d Luciferase reporter analysis of miR-382-5p mimics/ mimics-NC co-transfected with either MCM5-3'UTR-wt or MCM5-3'UTR -mut vector into 293 T cells. ${ }^{\star} p<0.05,{ }^{\star *} p<0.01$ 
a
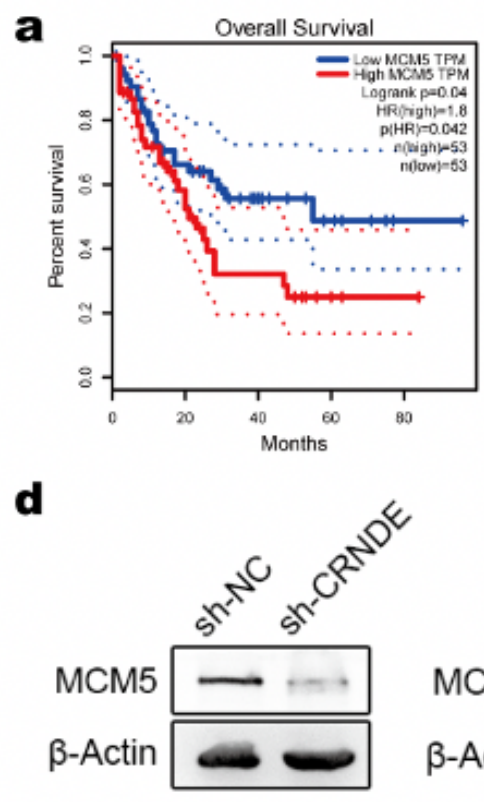

U937 b

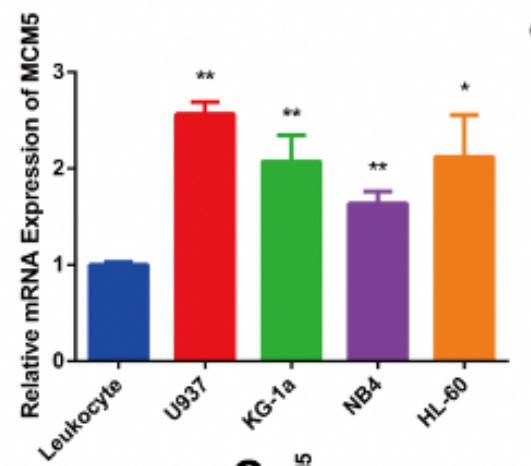

e

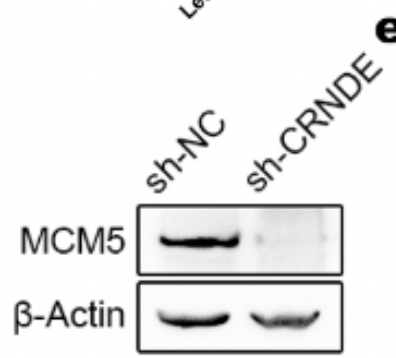

KG-1a

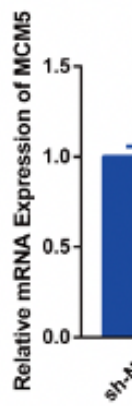

c

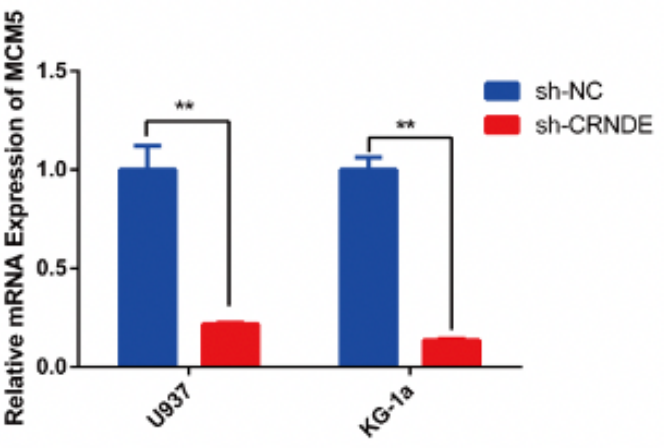

U937
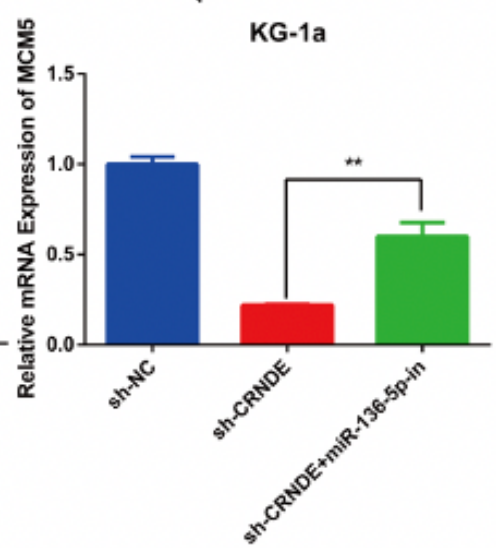

sh-CRNDE - + +

miR-136-5p-in

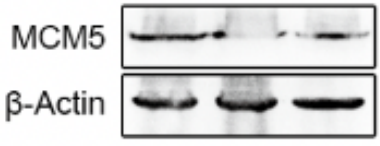

KG-1a

\section{Figure 6}

CRNDE regulates MCM5 through sponging miR-136-5p. a The overall survival curves of MCM5 in AML patients from GEPIA analysis. b MCM5 expression in AML cell lines (U937 and KG-1a) was detected by QPCR. c QPCR assay was used to detect the expression of MCM5 after knocking down CRNDE. $d$ Western blot was used to detect the expression of MCM5 protein after knocking down CRNDE. e-f Rescue assay was performed to verify that the effects could be reversed by miR-136-5p inhibitors at both mRNA and protein levels. ${ }^{*} p<0.05,{ }^{*} p<0.01$ 


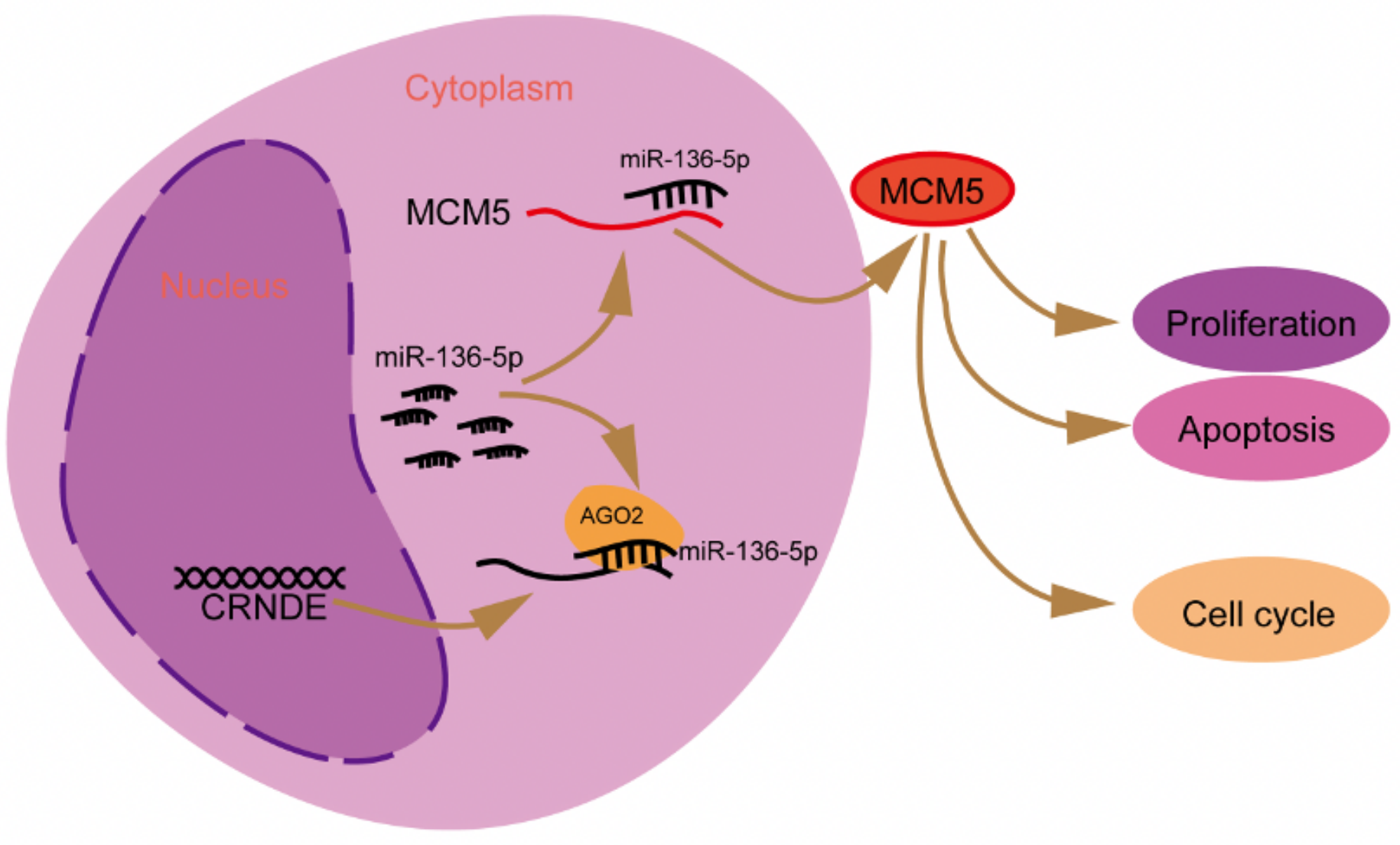

Figure 7

The possible modulating mechanism of CRNDE in AML cells by sponging miR-136-5p. 